\title{
Prime time: Fatigue and set effects in the perception of reversible figures
}

\author{
GERALD M. LONG, THOMAS C. TOPPINO, and GREGORY W. MONDIN \\ Villanova University, Villanova, Pennsylvania
}

\begin{abstract}
Subjects viewed unambiguous versions of both stationary and rotating Necker cube illusions for varying durations prior to the presentation of the standard ambiguous figure. In each case, the subjects were more likely to report the ambiguous figure to be (1) in the same configuration as that of the preceding prime following brief preexposure periods and (2) in the opposite configuration from that of the preceding prime following long preexposure periods. In addition, the number of reversals of the figure during the test period was also strongly related to the duration of the preexposure period, with progressively fewer reversals reported following longer preexposure periods. The results are interpreted as revealing the concurrent roles of "set" effects in the brief preexposure conditions and neural fatigue effects in the long preexposure conditions. Furthermore, the ability of the proposed two-process model to integrate the myriad of empirical effects in the reversible-figure literature is emphasized.
\end{abstract}

Reversible figures have generated extensive research interest because their curious multistable character is thought to provide insight into the functioning of the perceptual system. Unfortunately, the long-standing popularity of reversible figures in the literature does not reflect agreement among researchers concerning the precise nature of what this functioning might be. Within the current literature, there are investigators who favor cognitive processes such as learning and problem solving, which may influence illusory reversals in a "top-down" manner (e.g., Girgus, Rock, \& Egatz, 1977; Gregory, 1974; Rock, 1975). There are also investigators who favor the more passive or "bottom-up" processes of neural fatigue and recovery among cortical structures as determiners of figural reversal (e.g., Nawrot \& Blake, 1989; Palmer \& Bucher, 1981; von Grünau, Wiggin, \& Reed, 1984).

These interpretive differences do not result from any lack of empirical effort. In fact, the two general classes of explanations of reversible figures have impressive empirical support that has taken several forms. For example, the role of cognitive processes would appear to be required by the findings that instructions to observers can significantly alter reversal rates of subsequently viewed figures (Hochberg \& Peterson, 1987; Liebert \& Burk, 1985; Pelton \& Solley, 1968). The demonstrations that the presence of a distractor task slows the rate of perceived reversals (Reisberg, 1983; Reisberg \& O'Shaughnessy, 1984) and that, unless observers know that the figures being viewed are reversible, they may fail to perceive any reversals at all (Girgus et al., 1977) are also difficult to

The results contained in this article were presented in November 1991 at the meeting of the Psychonomic Society in San Francisco. Reprint requests should be sent to G. M. Long, Department of Psychology, Villanova University, Villanova, PA 19085. accommodate within a model dependent solely on lower level processes. In contrast, the role of localized neural processes is strongly implicated in several empirical findings. For example, two or more simultaneously presented reversible figures can be perceived in opposite configurations at the same time (Long \& Toppino, 1981; Long, Toppino, \& Kostenbauder, 1983) - not a finding readily reconciled with a hypothesis-testing conceptualization of the basis for reversals. Several studies have also demonstrated that selectively adapting the observer to particular stimulus configurations that should fatigue relevant neural structures (i.e., sharing the same localized neural channels) significantly alters both the likelihood of a particular configuration's being reported and the reversal rate between configurations of a reversible figure (e.g., Nawrot \& Blake, 1989; Toppino \& Long, 1987; von Grünau et al., 1984).

Given the wealth of empirical support for the two classes of underlying processes, it would appear increasingly likely that any ultimate explanation of reversible figures will require the integration of both types of processes. And indeed, several investigators over the past decade have argued for some type of hybrid theory of figural reversal that incorporates multiple processes (e.g., Hochberg \& Peterson, 1987; Long et al., 1983; Palmer \& Bucher, 1981). The present research represents an attempt to extend this conceptual approach by demonstrating in the same study the concurrent roles of cognitive and sensory factors in reported reversals of reversible figures. In addition, this work was an attempt to reconcile apparent contradictions in the empirical literature that have made integration of the literature most difficult. Specifically, we focused on a particular class of finding obtained with reversible figures that historically has been interpreted at one time as unambiguous evidence for cognitive effects and at other times as unambiguous evidence 
for sensory effects. Those targeted findings involve the effect of prior presentation of (or preexposure to) some stimulus array on the subsequent perception of a reversible figure by an observer.

An extremely popular demonstration in many introductory or perceptual texts is the impact of "set" on the perception of a reversible figure. Following the original work of Leeper (1935) with Boring's wife/mother-in-law figure or that of Fisher (1967) with the man/girl figure, the typical demonstration involves the initial presentation of an unambiguous version of the figure and the subsequent presentation of the reversible figure. There is a clear tendency to perceive the latter figure in the same configuration as that of the previously viewed figure, and this is described as a powerful demonstration of the role played by expectancy in the interpretation of incoming information (see Epstein \& Rock, 1960; Haber, 1966).

Unfortunately, in several ostensibly very similar (but generally less known) studies, the opposite result has been obtained, and this has been interpreted as providing clear support for the role of neural fatigue in the perception of such figures. For example, Harris (1980), extending some earlier work by Virsu (1975), reported that observers exposed to an unambiguous version of Shroeder's staircase tended to perceive the subsequently presented reversible version of that figure in the opposite perspective. Similarly, von Grünau et al. (1984) found that initially exposing observers to an unambiguous array of squares produced an increase in the reports of a Necker cube-like figure in the opposite orientation. More recently, Nawrot and Blake (1989) reported that prior exposure to one clear direction of motion resulted in an ambiguous-motion display appearing to move in the opposite direction. As Toppino and Long (1987; Long, 1988) have discussed in detail, this class of studies borrows directly from the selective-adaptation research which has sought (quite successfully) to identify the nature and functioning of the neural channels that compose our visual system.

In the present study, we sought to reconcile the older findings that so clearly implicated set effects with the later findings that have been so consistent with the known functioning of neural structures in the visual system. We hypothesized that a critical moderating variable in this type of work is the duration of the prior exposure. Specifically, we proposed that brief exposures to a particular configuration may "prime" the system in a manner consistent with cognitive set effects, essentially increasing the accessibility of similar perceptual organizations. Hence, following brief exposures to the unambiguous figure, the observer is more likely to perceive the subsequently presented reversible figure in the same configuration. With extended viewing of the unambiguous figure, however, the underlying cortical structures become fatigued, rendering them less available than the structures underlying the alternative organization of the reversible figure. Thus, as exposure to the unambiguous figure lengthens, the priming effect is reduced and eventually reversed by the influence of progressively increasing fatigue. That is, the observer becomes increasingly likely to exhibit an adaptation effect in which the subsequently presented reversible figure is perceived in a different configuration. ${ }^{1}$

In addition, we predicted that different durations of a preexposure interval would have a pronounced effect on another aspect of an observer's experience with reversible figures. It has been reported in several studies that the number of phenomenal reversals of reversible figures increases over a viewing interval (e.g., Babich \& Standing, 1981; Brown, 1955; Cohen, 1959; Toppino \& Long, 1987). However, different durations of prior exposure to an unambiguous version of a reversible figure will further influence the levels of reversals experienced during a viewing interval. Specifically, the different levels of neural fatigue produced by brief versus long preexposure periods are theorized to produce very different rates of phenomenal reversal. Very simply, the longer preexposure durations, which are predicted to produce appreciable fatigue in one class of underlying neural structures, are expected to result in significantly lower reversal rates in the testing period. This is attributed to the temporary "unavailability" of the structures necessary for one of the perceptual alternatives of the reversible figure. In contrast, the shorter preexposure durations, which are predicted to have little effect on the balance between the underlying neural components, are expected to produce relatively higher reversal rates in the testing period.

These demonstrations should have two important consequences. First, they should serve to reconcile apparent contradictions in the reversible-figure literature, rendering eventual integration of that literature more manageable. Second, such results should also provide impressive evidence for some version of the multiprocess model of reversible figures.

\section{METHOD}

\section{Subjects}

A total of 84 undergraduate college students at Villanova University were recruited as volunteers for participation in this study. Each subject was run in individual 60 -min sessions. All of the subjects received credit toward partial fulfillment of the requirements of an introductory psychology course. One volunteer was omitted because of self-reported poor vision in his right eye, and a second volunteer was omitted because he was unable to experience reversals with the rotating stimulus during practice.

\section{Apparatus}

There were two conditions (stationary and rotating) in the experiment, each requiring a different version of a reversible figure. The stationary figure was an ambiguous, two-dimensional (2-D) drawing of a Necker cube-like figure that had previously been used in a study done by von Grünau et al. (1984). The figure consisted of five overlapping $8 \times 8 \mathrm{~cm}$ squares and was drawn in black ink on a $20 \times 25 \mathrm{~cm}$ white posterboard background. The unambiguous priming stimuli were of identical size and make-up, but they were drawn to represent either the right or the left orientation. Examples of the stationary figures, both ambiguous and unambiguous, are shown in Figure 1. The ambiguous cube for the rotating condition was a $3-D$ skeleton cube measuring $8 \times 8 \times 8 \mathrm{~cm}$ and constructed with 3 -mm balsa wood sticks painted black. A solid $8 \times 8 \times 8 \mathrm{~cm}$ 


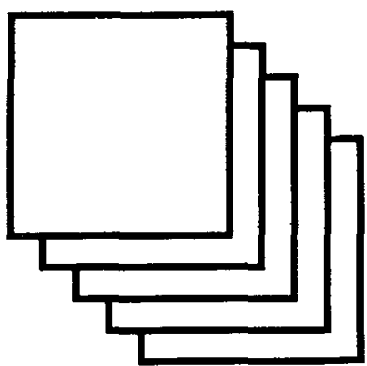

a

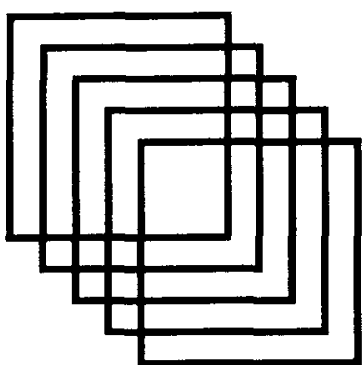

b

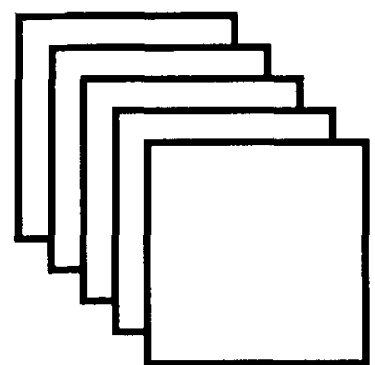

C

Figure 1. The Necker cube-like figure (b) composed of overlapping squares used as the stationary reversible figure in the present work, and its two possible interpretations (a and c) that were used as unambiguous preexposure figures.

cube, white with black edges, served as an unambiguous prime. Each cube rotated at a constant speed of $10 \mathrm{rpm}$. No fixation points were used in either the stationary or the rotating condition.

The figures were placed in front of a white background and were illuminated by a 100 -W light bulb positioned $0.5 \mathrm{~m}$ above the figures. The luminance of the white background was $85.7 \mathrm{~cd} / \mathrm{m}^{2}$. In the 3-D cube condition (i.e., the rotating condition), this background served as the background for the black cube. In the 2-D condition with overlapping squares (i.e., the stationary condition), the white posterboard backing for the black outline squares had a luminance of $85.7 \mathrm{~cd} / \mathrm{m}^{2}$. A sliding panel placed in front of the figures made it possible for the experimenter to show the subject one version of a figure at any given time. An Ealing electronic shutter that was positioned in front of the subject's right eye controlled the amount of time that subjects were able to view the figure (see below). The subjects' heads were stabilized with a head- and chinrest directly behind the shutter, and all subjects viewed the figures monocularly with the right eye from a distance of $2 \mathrm{~m}$. The number of reversals experienced by the subjects was recorded on an electronic counter that was advanced by the subject's depressing a telegraph key with his/her right index finger.

\section{Procedure}

Initially, 62 subjects were randomly assigned to either the stationary condition or the rotating condition. After a subject's assignment to one of the stimulus conditions, a drawing of an ambiguous Necker cube was used by the experimenter to explain and demonstrate the reversible quality of reversible figures. When the subject was able to acknowledge both possible orientations and to experience reversals with the demonstration drawing, the explanation and demonstration were repeated with the ambiguous and unambiguous figures appropriate for the respective condition. The second demonstration continued until three reversals were reported. Hence, the subject was familiarized with all stimuli prior to beginning any data collection.

The subjects were then "talked through" a practice trial, during which they were given instructions on when to report the initial orientation, when the shutter would open and close, and when to report reversals. The first practice trial lasted until the subject reported two reversals after the initial perception. A second practice trial, which was identical to the actual experimental trials, followed. A 2-min rest period followed this initial viewing to allow potential fatigue effects to dissipate.

An experimental trial began with the presentation of the unambiguous preexposure stimulus for the specified duration (see below), immediately followed by presentation of the ambiguous test stimulus. The subjects were instructed to report their initial perception of the orientation of the test stimulus: either front face "down-right" or "left-up" in the stationary condition or rotating "left" or "right" in the rotating condition. In addition, after the verbal report of their initial perception, the subjects reported reversals of perspective for a 30-sec test period by depressing a telegraph key each time a reversal was perceived. The experimenter recorded the number of reversals reported in each $10-\mathrm{sec}$ interval of the $30-\mathrm{sec}$ test period.

To provide a wide array of preexposure durations, and on the basis of unpublished pilot work, the lengths of the preexposure durations were chosen to be $0,1,5,15,30,60$, and $90 \mathrm{sec}$. The condition of 0-sec preexposure, in which subjects were presented with only the test stimulus, served as a control condition and provided a baseline for perceived reversals without any prior preexposure period. Within the 60-min session, each subject was presented with a uniquely randomized sequence of 14 trials, which consisted of 2 trials with the 0 -sec preexposure condition and 2 trials with each of the six nonzero preexposure conditions. For the latter conditions, one of the 2 trials at each duration involved one of the possible nonambiguous configurations (e.g., Figure 1a), and the other trial at that duration involved the alternative nonambiguous configuration (e.g., Figure 1c). There were 2-min rest periods between all 14 trials to allow for dissipation of potential fatigue effects. Research has indicated that this duration of a rest period should permit the dissipation of typical neural fatigue effects from even a long 2-min preexposure period (e.g., Magnussen \& Johnsen, 1986).

\section{RESULTS}

\section{Initial Perception After Adaptation}

The first measure of interest in the present study involved the effect of the unambiguous stimulus duration on the initial perception of the subsequent ambiguous test stimulus. For the present purposes, a same response was recorded when an observer reported an initial configuration of the ambiguous test stimulus that matched the configuration of the previously viewed preexposure stimulus. Note that a maximum of two same responses per subject was possible for each preexposure duration, because the subject received two trials with each duration. Hence, the number of same responses recorded per duration for each subject was 0,1 , or 2 . For each condition (i.e., stationary and rotating illusion), a one-way withinsubject analysis of variance (ANOVA) was performed on the mean number of same responses over the six pre- 
exposure durations (i.e., 1-, 5-, 15-, 30-, 60-, and 90sec preexposure durations). (The 0 -sec preexposure condition was omitted from this initial analysis because same or different responses were conceptually impossible without a preceding unambiguous stimulus.) In the stationarysquares condition, a significant main effect for duration was obtained $[F(5,150)=2.73, p=.02]$. Figure 2 presents the mean number of same responses as a function of preexposure duration for the stationary illusion condition.

Planned comparisons reveal highly significant differences between the means at 1 and $90 \mathrm{sec}(p<.001)$, 1 and $60 \sec (p<.05)$, and 5 and $90 \sec (p<.025)$. To assist in determining whether the pattern of fewer same responses at the longer durations continued beyond $90 \mathrm{sec}$, an additional 10 subjects from the same subject pool were run under identical conditions, but with preexposure durations of $60,90,120$, and $150 \mathrm{sec}$. The mean number of same responses obtained with this second group of subjects are graphically displayed with a dotted line in Figure 2. A planned comparison revealed a significant difference between the means at 60 and $150 \sec (p<.025)$, and between the means at 90 and $150 \mathrm{sec}(p<.10)$.

An important prediction of this work involved the " direction" of the preexposure effect across the different preexposure durations. Additional formal planned compari-

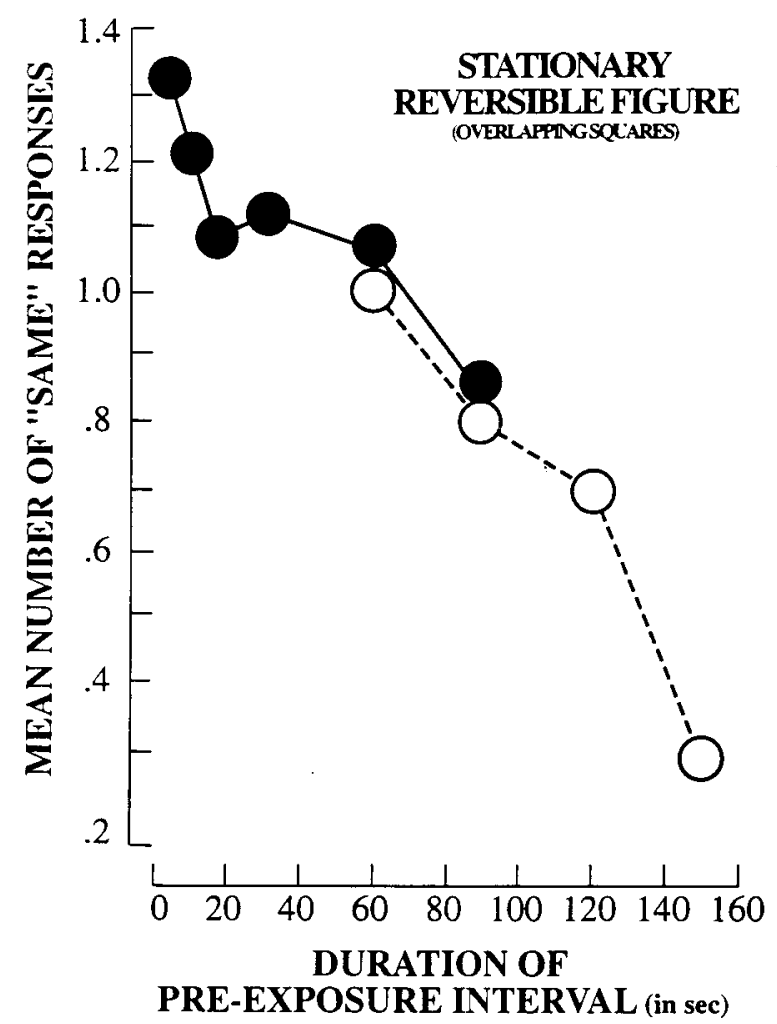

Figure 2. Mean number of initial reports of the stationary reversible figure's configuration that were the same as that of the preceding unambiguous stimulus, plotted as a function of the preexposure duration. (The dashed line presents the results from an additional group of 10 subjects tested over a different range of preexposure durations.)

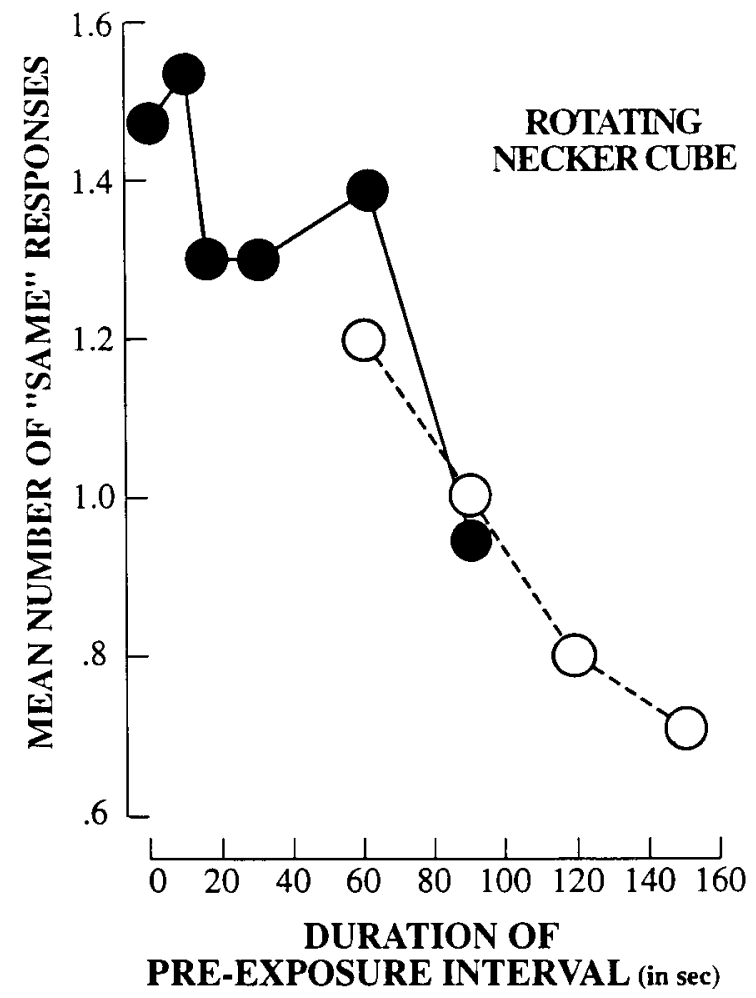

Figure 3. Mean number of initial reports of the rotating Necker cube's direction of rotation that were the same as that of the preceding unambiguous stimulus, plotted as a function of the preexposure duration. (The dashed line presents the results from an additional group of 10 subjects tested over a different range of preexposure durations.)

sons were performed between the obtained means in each group of subjects and the expected mean of 1.0. (A mean of 1.0 represents the expected mean of same responses for two trials, based on chance responses to an ambiguous stimulus with two possible configurations.) The a priori comparisons in the stationary condition revealed that the mean at the 1-sec duration was significantly greater than the expected mean of $1.0[t(30)=3.18, p<.005]$; the mean at the 150-sec duration was significantly less than the expected mean of $1.0[t(9)=4.67, p<.005]^{2}$

For the rotating-cube condition, a significant effect for duration was also obtained $[F(5,150)=5.13, p<.001]$. The mean number of same responses as a function of prime duration in the rotating condition is shown in Figure 3.

Planned comparisons revealed highly significant differences between the means at 1 and $90 \mathrm{sec}(p<.001)$, 5 and $90 \sec (p<.001)$, and 60 and $90 \sec (p<.001)$. The means for the 15- and 30-sec durations, which were identical, differed significantly from the 90-sec mean $(p<$ .01 ). As in the stationary condition, an additional 10 subjects from the same subject pool were run with preexposure durations of $60,90,120$, and $150 \mathrm{sec}$. The mean number of same responses as a function of preexposure duration are shown graphically with a dotted line in Figure 3. Planned comparisons between the means obtained for this second group of subjects revealed significant dif- 


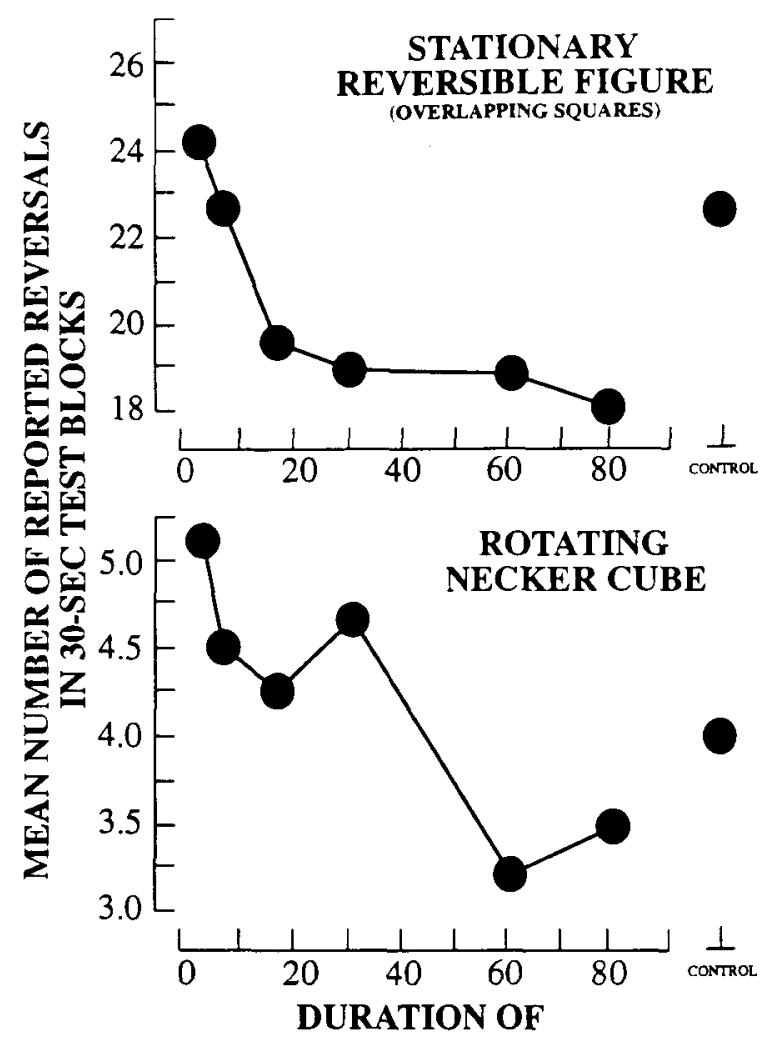

PRE-EXPOSURE INTERVAL (in sec)

Figure 4. Mean number of reversals reported for the stationary reversible figure (upper portion) and for the rotating Necker cube (lower portion) during the 30-sec test interval, plotted as a function of the preexposure duration.

ferences between the means at 60 and $150 \sec (p<.05)$, and between the means at 60 and $120 \sec (p<.10)$. Additional comparisons between the means for the two extreme preexposure conditions and the expected mean of 1.0 revealed the mean for the $1-\sec$ condition to be significantly greater than $1.0[t(30)=4.80, p<.0001]$ and the obtained mean at $150 \mathrm{sec}$ to be significantly less than the expected mean of $1.0[t(9)=2.00, p<.05]$.

\section{Reversal Rates After Preexposure}

Another major focus of the present work was the effect of preexposure periods of different duration on the figural reversal rates in the subsequent testing period. Figure 4 presents the mean number of reversals reported in the 30-sec testing period as a function of the duration of the preexposure period for the stationary and rotating conditions. In both cases, a decreasing function is clearly evident. For formal analysis, the mean number of reversals was examined as a function of preexposure duration for the three 10-sec blocks in the testing period. A 3 (10sec blocks) $\times 7$ (preexposure durations) within-subject ANOVA on the reversals in the stationary-cube condition revealed significant main effects of blocks $[F(2,60)=$ $6.17, p<.005]$ and duration $[F(6,180)=8.69, p<$ $.0001]$. These effects are readily apparent in Figure 5, which presents the mean number of reversals separately for the three 10 -sec blocks of the test interval. It can be seen that the number of reversals generally increases over the three 10-sec blocks of the test period. The small but significant block $\times$ duration interaction $[F(12,360)=$ $2.04, p<.05$ ] results from the fact that the overall positive effect of viewing block is only clearly present for the five longer viewing durations $(p<.05$ in each case between the first and third 10-sec block by planned comparisons). Also evident in Figure 5 is the fact that more reversals tend to occur following the briefer as opposed to the longer preexposure periods. Within each of the three 10 -sec blocks, the mean number of reversals for the 1and 5-sec durations differed significantly from the mean number of reversals for the 30-, 60-, and 90-sec durations ( $p<.01$ in each case).

A comparable series of analyses that was undertaken for the reversals in the rotating-cube condition is depicted in Figure 6. The 3 (blocks) $\times 7$ (duration) within-subject ANOVA revealed once again significant main effects of block $[F(2,60)=9.76, p<.001]$ and duration $[F(6,180)$ $=2.17, p<.02]$. The mean number of reversals increased over the three 10 -sec blocks of the testing period and decreased as the preexposure duration increased. The block $\times$ duration interaction was not significant $(F<$ 1.0). Further probing of the mean differences indicates

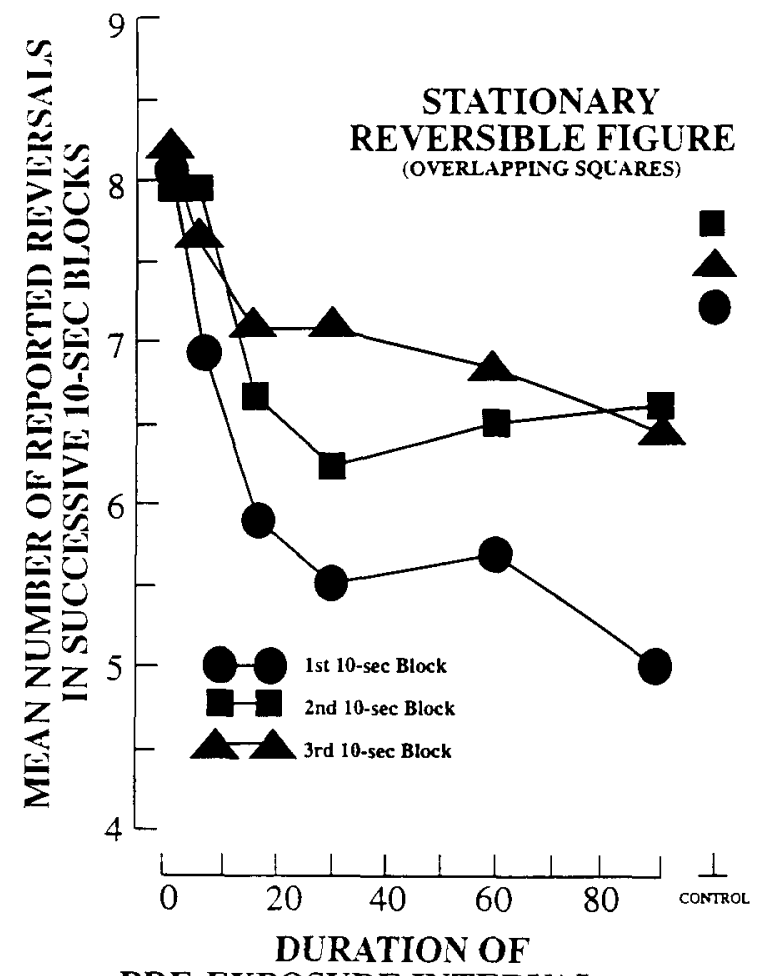

PRE-EXPOSURE INTERVAL (in sec)

Figure 5. Mean number of reversals reported for the stationary reversible figure during each of the successive 10-sec blocks comprising the test interval, plotted as a function of the preexposure duration. 


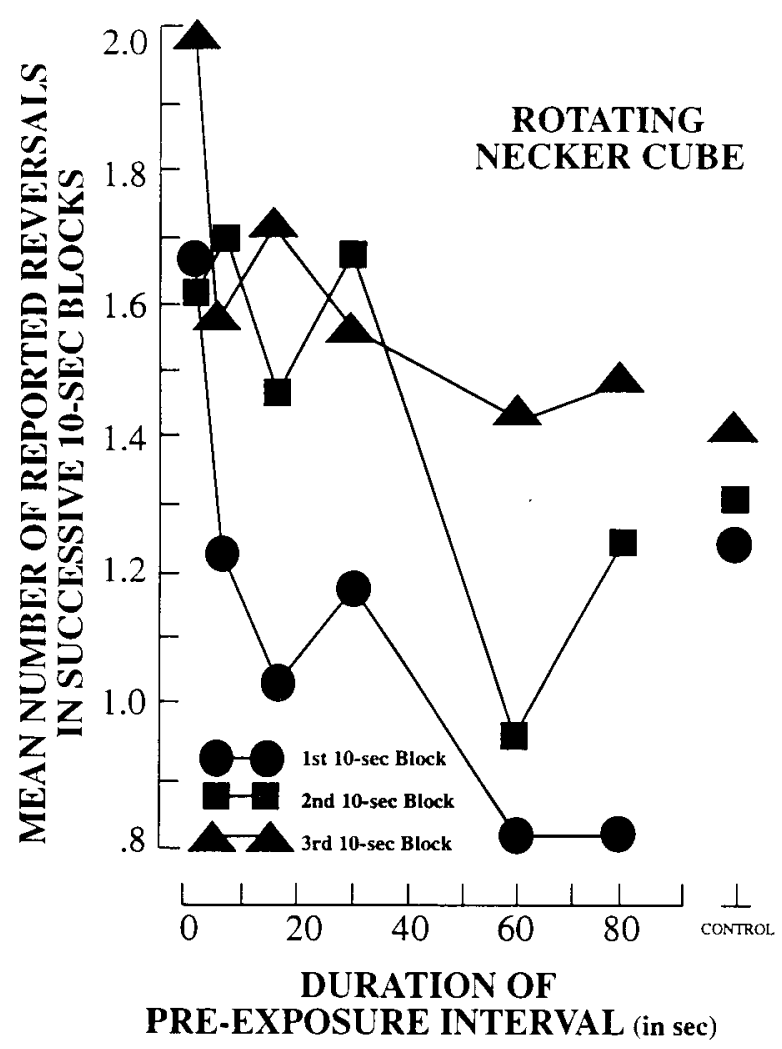

Figure 6. Mean number of reversals reported for the rotating Necker cube during each of the successive 10-sec blocks comprising the test interval, plotted as a function of the preexposure duration.

that, within each of the three 10 -sec blocks, there were significant differences between the mean for the $1-\sec$ duration and the means for the $60-\sec (p<.05)$ and $90-\sec$ $(p<.10)$ durations.

\section{DISCUSSION}

The purpose of the present study was to demonstrate the relative roles of cognitive and fatigue processes in the perception of reversible figures and thereby reconcile two classes of results that have traditionally been treated as favoring mutually exclusive models of the reversal process. Two hypotheses were offered regarding the effects of varying the duration of the preexposure period prior to the viewing of a reversible figure. The first prediction stipulated that the likelihood with which an observer would report a reversible figure to be in the same configuration as that of an unambiguous figure presented during a preceding preexposure period would decrease as the duration of that preexposure period increased. A related prediction was that, following brief preexposure periods, observers would be more likely to report the reversible figure in the same configuration as that of the unambiguous figure than would be expected by chance. And, following long preexposure periods, observers would be more likely to report the reversible figure to be in the configuration opposite that of the unambiguous figure than would be expected by chance. The results depicted in Figures 2 and 3 are clearly supportive of this first set of predictions for both the stationary and the rotating illusions. ${ }^{3}$

The second prediction stipulated that the reversal rates obtained over the testing period, in addition to a general increase within the period, would also be altered by the duration of the preceding preexposure period. Specifically, relatively few reversals were expected following long preexposure periods, and relatively frequent reversals were expected following brief preexposure periods. The results depicted in Figures 4, 5, and 6 are clearly supportive of this second set of predictions for both the stationary and the rotating illusions.

The obtained pattern of results is thought to reflect two very different types of processes in the perception of reversible figures. Brief exposure of an unambiguous version of a reversible figure is believed to "prime" the individual to interpret the subsequent reversible figure in the same configuration as that of the prime. This is attributed to "set" effects, as was argued in several of the oftcited older studies in the reversible-figure literature (e.g., Botwinick, 1961; Bugelski \& Alampay, 1961; Leeper, 1935; Fisher, 1967). Longer exposures to the same unambiguous version of a reversible figure are believed to produce appreciable fatigue in the underlying neural structures, as has been argued in several more recent studies in the reversible-figure literature (e.g., Nawrot \& Blake, 1989; Petersik, Shepard, \& Malsch, 1984; von Grünau et al., 1984). This fatigue is hypothesized to establish an imbalance in the underlying structures. Consequently, upon presentation of the reversible figure, the alternate neural channels that were not fatigued during the preexposure period are favored. As a result, there is a greater likelihood that the observer will report the configuration opposite that of the subsequent reversible figure, and there is also a restriction of the possible reversal rates during the testing period while the fatigue within those channels dissipates. This conceptualization is borrowed directly from the selective-adaptation paradigm that has become so popular in the spatial vision literature (cf. DeValois \& DeValois, 1988; Frisby, 1980).

Numerous results in the literature reinforce the explanatory value of the two-process model, which posits qualitatively different processes underlying particular viewing conditions with reversible figures. Consider recent experiments in which eye movements have been controlled with a fixation point and in which adaptation effects have been obtained when the preexposure stimulus and the subsequent reversible figure are presented to the same region of the visual field but not when these stimuli are presented to markedly different locations within the visual field (Toppino \& Long, 1987; von Grünau et al., 1984). Although such findings can readily be explained by a stimulus-driven model based on the fatigue of localized neural structures, they are not easily explained by a model that attributes the perception of reversible figures solely to top-down 
cognitive processes. The situation is reversed, however, when one considers the older studies in which reversible figures were employed in the investigation of set effects in perception. Because of the presumed cognitive nature of such effects, the set-inducing stimuli were often very different physically (i.e., in terms of shared features) from the reversible figures themselves. For example, Bugelski and Alampay (1961) successfully presented figures of animals (e.g., dog, cat, duck, etc.) to set the observer for the "rat" interpretation of the rat/man figure and presented figures of people (e.g., boy, girl, baby, etc.) to set the observer for the "man" interpretation. Clearly, a stimulus-driven model based on neural fatigue can make no obvious predictions in such a situation; but a conceptually driven model emphasizing cognitive organization would have little difficulty with such results.

Finally, we wish to stress the point that the empirical findings in the present work, as well as the apparent utility of the multiprocess model of figural reversal, most strongly impress us with the value of reversible figures as research tools. And these tools may be applicable in a much broader manner than has been appreciated in the past. At the theoretical level, the multiple configurations of such well-known figures as the Necker cube serve as a useful metaphor for the multiple processes that underlie our perceptual abilities. The overly narrow conceptualizations of much of the early work has been found to be too limiting. At the practical level, figural reversals may reflect in an especially powerful way both stimulusdriven and conceptually driven processes and may therein serve to reveal the nature and interaction of these two levels of processing.

\section{REFERENCES}

BABich, S., \& STANDING, L. (1981). Satiation effects with reversible figures. Perceptual \& Motor Skills, 52, 203-210.

Borwinick, J. (1961). Husband and father-in-law-A reversible figure. American Joumal of Psychology, 74, 312-313.

Brown, K. T. (1955). Rate of apparent change in a dynamic reversible figure as a function of observation time. American Journal of Psychology, 68, 358-371.

Bugeiski, B. R., \& Alampay, D. A. (1961). The role of frequency in developing perceptual sets. Canadian Journal of Psychology, 15, 205-211.

CoHEN, L. (1959). Rate of apparent change of a Necker cube as a function of prior stimulation. American Journal of Psychology, 72, 327-344.

DeValois, R. L., \& DeVAlors, K. K. (1988). Spatial vision. New York: Oxford.

EPSTEIN, W., Rock, I. (1960). Perceptual set as an artifact of recency. American Journal of Psychology, 73, 214-228.

Fisher, G. H. (1967). Preparation of ambiguous stimulus materials. Perception \& Psychophysics, 2, 421-422.

FrisBY, J. P. (1980). Seeing: Illusion, brain and mind. New York: Oxford University Press.

Girgus, J. J., Rock, J., \& Egatz, R. (1977). The effect of knowledge of reversibility on the reversibility of ambiguous figures. Perception \& Psychophysics, 22, 550-556.

GREgory, R. L. (1974). Choosing a paradigm for perception. In E. C Carterette \& M. P. Friedman (Eds.), Handbook of perception: Vol. I.
Historical and philosophical roots of perception (pp. 255-283). New York: Academic Press.

Haber, R. N. (1966). Nature of the effect of set on perception. Psychological Review, 73, 335-351.

HarRIS, J. P. (1980). How does adaptation to disparity affect the perception of reversible figures? American Journal of Psychology, 93 , 445-457.

Hochrerg, J., \& Peterson, M. A. (1987). Piecemeal organization and cognitive components in object perception: Perceptually coupled responses to moving objects. Joumal of Experimental Psychology: General, 116, 370-380.

LEEPER, R. W. (1935). A study of a neglected portion of the field of leaming: The development of sensory organization. Jourmal of Genetic Psychology, 46, 41-75.

Liebert, R. M., \& BURK, B. (1985). Voluntary control of reversible figures. Perceptual \& Motor Skills, 61, 1307-1310.

LoNG, G. M. (1988). Selective adaptation vs. transfer of decrement: The conjoint effects of neural fatigue and perceptual learning. Perception \& Psychophysics, 43, 207-209.

LoNG, G. M., \& TOPPINO, T. C. (1981). Multiple representations of the same reversible figure: Implications for cognitive decisional interpretations. Perception, 10, 231-234.

Long, G. M., Toppino, T. C., \& Kostenbauder, J. F. (1983). As the cube turns: Evidence for two processes in the perception of a dynamic reversible figure. Perception \& Psychophysics, 34, 29-38.

MAGNuSSEN, S., \& JOHNSEN, T. (1986). Temporal aspects of spatial adaptation: A study of the tilt aftereffect. Vision Research, 25, 661-672.

NAWROT, M., \& BLAKE, R. (1989). Neural integration of information specifying structure from stereopsis and motion. Science, 244, 716-718.

Palmer, S. E., \& Bucher, N. M. (1981). Configural effects in perceived pointing of ambiguous triangles. Joumal of Experimental Psychology: Human Perception \& Performance, 7, 88-114.

Pelton, L. H., \& Solley, C. M. (1968). Acceleration of reversals of a Necker cube. American Journal of Psychology, 81, 585-588.

Petersik, J. T., Shepard, A., \& Malsch, R. (1984). A threedimensional motion aftereffect produced by prolonged adaptation to a rotation simulation. Perception, 13, 489-497.

REISBERG, D. (1983). General mental resources and perceptual judgments. Joumal of Experimental Psychology: Human Perception \& Performance, 9, 966-979.

Reisberg, D., \& O'Shaughnessy, M. (1984). Diverting subjects' concentration slows figural research. Perception, 13, $461-468$.

Rock, I. (1975). An introduction to perception. New York: Macmillan.

Toppino, T. C., Long, G. M. (1987). Selective adaptation with reversible figures: Don't change that channel. Perception \& Psychophysics, 42, 37-48.

VIRSU, V. (1975). Determinants of perspective reversals. Nature, 257, 786-787.

von Grünau, M. W., Wiggin, S., \& Reed, M. (1984). The local character of perspective organization. Perception \& Psychophysics, $35,319-324$.

\section{NOTES}

1. Unfortunately, it is not possible to determine the effects of different preexposure durations by comparing the studies in which different results have been obtained with this same basic paradigm. The studies differ on a number of potentially important dimensions, including the particular reversible figure chosen, the presence of a fixation point, the instructions to subjects, and the precision of their procedural descriptions. All of these factors render direct comparisons problematic.

2 . The rationale detailed in the introduction permitted us to make firm predictions only for the most extreme preexposure conditions. The precise duration for which the effect of the preexposure stimulus would shift from that of a priming stimulus to that of an adaptation stimulus was not specified. Hence, we have only compared the obtained means for the most extreme conditions ( 1 and $150 \mathrm{sec}$ ) with the expected mean of 1.0 . 
3. One anonymous reviewer noted that we may have incorporated a potential confound into our procedures. Specifically, the brief exposure conditions involved shorter overall trial length than did the long preexposure conditions. This raises the possibility that duration of the trial per se rather than duration of the type of exposure (as we posit) was the critical variable. However, we are unable to reconcile the obtained pattern of results with the effect of a nonspecific "trials' length" factor. Remember that for each preexposure duration, the subject was tested twice-once with each of the two possible unambiguous configurations of the ambiguous figure (i.e., the two configurations were counterbalanced at each duration). Hence, to produce the obtained results, this trials' length factor would have to have been sensitive to the type of configuration presented, biasing the subject differently for the two configurations. This seems highly unlikely.

(Manuscript received August 26, 1991; revision accepted for publication May 19, 1992.)

\section{Fifth International Conference on Vision in Vehicles University of Glasgow, Scotland September 9-11, 1993}

The Applied Vision Association in conjunction with the Ergonomics Society and the Association of Optometrists announce the Fifth International Conference on Vision in Vehicles. This will be held at the Kelvin Conference Centre, University of Glasgow, Scotland, September 9-11, 1993. This multidisciplinary conference will provide an international forum for the exchange of information on current research encompassing all aspects of vision and its relationship to vehicle design. This includes both the internal and external design of the vehicle as well as the perceptual and cognitive capabilities and limitations of vehicle controller.

The scope of the conference will cover all types of vehicles where the task involves monitoring both in-vehicle displays and the surrounding visual environment in both day and night-time conditions. The conference will therefore be of interest to vehicle and instrument designers, psychologists, ergonomists, ophthalmologists, ophthalmic opticians, optometrists, highway engineers, traffic planners, lighting engineers, legislators, and anyone concerned with safety in vehicles.

Further information about the conference can be obtained from: VIV5, Academic Radiology, University Hospital, Queens Medical Centre, Nottingham NG7 2UH, U.K. (telephone, 44-0-602-709442; Fax, 44-0-602-709140). 